\title{
QUESTIONÁRIO DE SINTOMAS NA DOENÇA DO REFLUXO GASTROESOFÁGICO
}

\author{
Fernando FORNARI ${ }^{1}$, Antônio Carlos GRUBER ${ }^{2}$, Antônio de Barros LOPES ${ }^{3}$, \\ Dileta CECCHETTI ${ }^{4}$ e Sérgio Gabriel Silva de BARROS ${ }^{1}$
}

RESUMO - Racional - Questionários estruturados são valiosos instrumentos para medir o impacto de doenças na qualidade de vida dos pacientes através do cálculo de um escore e são disponíveis tanto no exterior, quanto no Brasil. Entretanto, questionários baseados em sintomas para a doença do refluxo gastroesofágico não são disponíveis na língua portuguesa. Objetivo - Desenvolver e validar na língua portuguesa um questionário específico de sintomas na doença do refluxo gastroesofágico. Pacientes e Métodos - Traduziu-se o questionário de Velanovich, acrescentou-se uma questão para o sintoma "regurgitação" e adaptou-se o vocabulário à escolaridade média estimada da população estudada. A "validade de face" para cada pergunta foi avaliada por um painel multidisciplinar e um questionário QS-DRGE consensual construído. O questionário foi, então, respondido por pacientes com sintomas de refluxo gastroesofágico confirmado por pHmetria esofagiana prolongada. Mediu-se sua compreensão, o tempo necessário para respondê-lo, sua reprodutibilidade e o coeficiente de correlação com o escore de Johnson-DeMeester. Resultados - A "validade de face" foi referendada pelo painel e o questionário aplicado a 124 indivíduos, consecutivamente. A compreensão do questionário e o tempo menor do que 5 minutos para seu preenchimento foram observados em todos os indivíduos (100\%). A reprodutibilidade em 10 indivíduos, em duas ocasiões distintas, apresentou elevado coeficiente de correlação intraclasse $(0,833)$. A correlação com o escore de Johnson-DeMeester foi nula. Conclusão - O questionário de sintomas na doença do refluxo gastroesofágico apresentou "validade de face", fácil compreensão e rápida resposta em todos os indivíduos com excelente reprodutibilidade. A correlação com o escore de Johnson-DeMeester foi nula.

DESCRITORES - Refluxo gastroesofágico. Sinais e sintomas digestórios. Questionários.

\section{INTRODUÇÃO}

Questionários de qualidade de vida relacionada à saúde, genéricos ou específicos, têm sido valiosos instrumentos para mensurar a qualidade de vida pela ótica do paciente ${ }^{(4)}$. Questionários genéricos são usados junto à população geral com o intuito de estudar, de forma ampla, determinados "domínios" da área de saúde. São aplicáveis também aos mais variados estados de saúde, condições ou doenças ${ }^{(14)}$.

VELANOVICH et al. ${ }^{(10)}$ construíram e validaram uma escala baseada em sintomas típicos para a doença do refluxo gastroesofágico (DRGE), objetivando simplicidade de uso e sensibilidade aos efeitos terapêuticos (Quadro 1).

O escore é calculado pela soma dos algarismos assinalados e pode variar entre 0 e 45 ( 0 a melhor resposta e 45 , a pior). A última questão avalia a percepção do paciente quanto ao seu estado de saúde atual em seis níveis de satisfação.

No Brasil é desconhecida a existência desse tipo de instrumento para a abordagem de pacientes com DRGE.

O objetivo deste estudo foi desenvolver e validar um questionário para calcular o escore de sintomas na DRGE antes e após intervenções terapêuticas.

\section{PACIENTES E MÉTODOS}

Validação do questionário de sintomas na doença do refluxo gastroesofágico (QS-DRGE)

Foi desenvolvido um protocolo baseado em metodologia de tradução para a língua portuguesa, de questionário de qualidade de vida conhecido em língua inglesa ${ }^{(3)}$, já previamente utilizado. Os passos seguidos foram os seguintes:

1. tradução para a língua portuguesa por dois professores da língua inglesa, brasileiros;

2. consenso da primeira tradução adequando a linguagem à escolaridade média estimada da população brasileira estudada, realizado pelos autores gastroenterologistas do estudo;

3. versão do consenso para a língua inglesa por dois professores da língua inglesa, norte-americanos radicados no Brasil;

4. comparação desta tradução com a versão original;

5. adaptação do questionário original acrescentando-se uma questão para avaliar o sintoma "regurgitação" à versão em português.

Para avaliar as características psicométricas do QSDRGE, realizou-se teste de reprodutibilidade (concordância

\footnotetext{
Trabalho apresentado ao Programa de Pós-graduação: Ciências em Gastroenterologia da Faculdade de Medicina da Universidade Federal do Rio Grande do Sul para obtenção do grau de Mestre. ${ }^{1}$ Faculdade de Medicina da Universidade Federal do Rio Grande do Sul, Porto Alegre, RS.; ${ }^{2}$ Núcleo Motilidade Digestiva do Hospital Moinhos de Vento, Porto Alegre, RS.; ${ }^{3}$ Serviço de Gastroenterologia do Hospital de Clínicas de Porto Alegre, RS.; ${ }^{4}$ Universidade de Passo Fundo, RS.;

Endereço para correspondência: Dr. Fernando Fornari - Rua Paissandu, 488/802 - Centro - 99010-102 - Passo Fundo, RS. E-mail: nandofornari@turbo.com.br
} 
QUADRO 1 - Gastroesophageal reflux data sheet

\begin{tabular}{|c|c|c|c|c|c|c|}
\hline Questions about symptoms (circle one for each question) & \multicolumn{6}{|c|}{ Scale } \\
\hline 1. How bad is the heartburn? & 0 & 1 & 2 & 3 & 4 & 5 \\
\hline 2. Heartburn when lying down? & 0 & 1 & 2 & 3 & 4 & 5 \\
\hline 3. Heartburn when standing up? & 0 & 1 & 2 & 3 & 4 & 5 \\
\hline 4. Heartburn after meals? & 0 & 1 & 2 & 3 & 4 & 5 \\
\hline 5. Does heartburn change your diet? & 0 & 1 & 2 & 3 & 4 & 5 \\
\hline 6. Does heartburn wake you from sleep? & 0 & 1 & 2 & 3 & 4 & 5 \\
\hline 7. Do you have difficulty swallowing? & 0 & 1 & 2 & 3 & 4 & 5 \\
\hline 8. Do you have pain with swallowing? & 0 & 1 & 2 & 3 & 4 & 5 \\
\hline 9. If you take medications, does this affect your daily life? & 0 & 1 & 2 & 3 & 4 & 5 \\
\hline 10. How satisfied are you with your present condition? & very satisfied & satisfied & neutral & dissa-tisfied & very dissa-tisfied & incapaci-tated \\
\hline
\end{tabular}

0 , no symptoms; 1 , symptoms noticeable, but no bothersome; 2 , symptoms noticeable and bothersome, but not every day; 3 , symptoms bothersome every day; 4 , symptoms affect daily activities; and 5 , symptoms are incapacitating - unable to do daily activities (VELANOVICH et al. (99)

de respostas em dois momentos distintos, medida pelo coeficiente de correlação intraclasse), avaliação da compreensão através de percentual de respostas (acima de 90\%) e a rapidez para completar as respostas pelos pacientes (tempo menor que 5 minutos). A validade de face, que significa a adequação do questionário para atingir os seus objetivos, foi avaliada em dois momentos, através de painel multidisciplinar com 25 mestrandos, doutorandos e professores orientadores, médicos e nãomédicos na Disciplina Seminários de Pesquisa do Programa de PósGraduação: Ciências em Gastroenterologia da Universidade Federal do Rio Grande do Sul, Porto Alegre, RS. Calculou-se também a correlação do QS-DRGE com o escore composto de refluxo gastroesofágico ácido de JOHNSON-DeMEESTER ${ }^{(6)}$, cujo valor discriminativo considerado foi de 22,0. A questão de número 11 possui opções de repostas como variável nominal, sendo analisada à parte do escore.

\section{Aplicação do QS-DRGE}

Inicialmente foi explicado ao paciente que o questionário continha várias perguntas sobre o sintoma azia e este foi esclarecido como uma sensação de ardência retroesternal, podendo atingir a garganta. $\mathrm{O}$ questionário auto-aplicável foi, então, respondido espontaneamente pelos pacientes colocados a sós numa sala silenciosa. Quando havia dúvidas sobre alguma pergunta o autor lia a questão com voz pausada e no mesmo timbre. Postulou-se que após essa leitura se persistisse o não entendimento da questão, o paciente seria convidado a responder à próxima questão. A não resposta à qualquer pergunta implicaria na exclusão do indivíduo do estudo e registrar-se-ia a não-compreensão do QS. Definiu-se que o tempo de preenchimento em até 5 minutos seria considerado rápido, sendo seu registro aferido pelo autor por relógio digital.

\section{Pacientes}

Foram estudados, consecutivamente, os pacientes encaminhados para realizar manometria e pHmetria esofagiana prolongada após a assinatura de consentimento informado previamente aprovado pela Comissão de Ética em Pesquisa da Instituição. Foram excluídos pacientes com analfabetismo primário ou funcional.

Para avaliar a reprodutibilidade do QS-DRGE, 10 pacientes com sintomas típicos ou atípicos de DRGE e refluxo gastroesofágico ácido, confirmado à pHmetria prolongada ${ }^{(6)}$,e que não haviam recebido tratamento farmacológico prévio, responderam ao questionário logo após a entrega do resultado da pHmetria. Após intervalo variável entre 10 a 15 dias, aqueles indivíduos voltaram a responder ao mesmo questionário. Os pacientes concordaram em não modificar a sua rotina diária e em não iniciar qualquer tratamento para a DRGE entre as duas ocasiões.
A seguir, todos os pacientes encaminhados ao laboratório, consecutivamente, responderam ao questionário QS-DRGE antes dos seus exames de pHmetria prolongada. Os que apresentaram refluxo ácido positivo (tempo total com $\mathrm{pH}$ inferior a 4,0 maior que $4,2 \%$ e/ou tempo na posição ortostática maior que $6,3 \%$ e/ou tempo na posição supina maior que $1,2 \%$ ) tiveram seus escores no QS-DRGE comparados aos obtidos pelo método de Johnson-DeMeester e avaliados também quanto à compreensibilidade do questionário QS-DRGE e ao período de tempo necessário (rapidez) para o seu preenchimento.

\section{Análise estatística}

A análise estatística descritiva foi utilizada na caracterização demográfica e clínica dos pacientes avaliados. O coeficiente de correlação intraclasse foi utilizado para avaliação da reprodutibilidade do questionário intra-observador. Seu resultado foi considerado significativo quando maior que 0,7 . A associação entre o escore composto de Johnson e DeMeester e o do QS-DRGE foi avaliada através da correlação linear simples. Os escores de Johnson e DeMeester e do QS-DRGE foram descritos através de média e desvio padrão. O nível de significância adotado foi de $\alpha=0,05$.

\section{RESULTADOS}

\section{Questionário de Sintomas}

Após três reuniões, os autores estabeleceram um consenso final da tradução e adaptação do questionário de Velanovich ao qual denominaram de Questionário de Sintomas na DRGE (QS-DRGE) (Quadro 2).

O escore é calculado pela soma dos algarismos assinalados pelos pacientes, podendo variar entre 0 e 50 ( 0 a melhor resposta e 50, a pior). A última questão avalia a percepção do paciente quanto ao seu estado de saúde atual em seis níveis de satisfação.

\section{Características dos Pacientes}

Pacientes com sintomas da DRGE e pHmetria prolongada positiva para refluxo gastroesofágico ácido foram incluídos (Tabela 1).

No período do estudo, 124 pacientes foram incluídos e os sintomas típicos (pirose e regurgitação) foram predominantes, mas sintomas atípicos ou extra-esofágicos estiveram representados no grupo estudado.

\section{Avaliação da Compreensão e do Tempo de Preenchi- mento do Questionário}

Todos os pacientes responderam a todas as questões num período de tempo inferior a 5 minutos. 
QUADRO 2 - Questionário de sintomas na doença do refluxo gastroesofágico (QS-DRGE)

\begin{tabular}{|c|c|c|c|c|c|c|}
\hline \multicolumn{7}{|c|}{ Perguntas sobre os sintomas (circule um número para cada questão): } \\
\hline 1. Quanto o incomoda a sua azia? & 0 & 1 & 2 & 3 & 4 & 5 \\
\hline 2. Sente azia quando está deitado? & 0 & 1 & 2 & 3 & 4 & 5 \\
\hline 3. Sente azia quando está em pé? & 0 & 1 & 2 & 3 & 4 & 5 \\
\hline 4. Sente azia após as refeições? & 0 & 1 & 2 & 3 & 4 & 5 \\
\hline 5. A azia altera seus hábitos de alimentação? & 0 & 1 & 2 & 3 & 4 & 5 \\
\hline 6. A azia acorda você durante o sono? & 0 & 1 & 2 & 3 & 4 & 5 \\
\hline 7. Você sente dificuldade para engolir? & 0 & 1 & 2 & 3 & 4 & 5 \\
\hline 8. Você sente dor ao engolir? & 0 & 1 & 2 & 3 & 4 & 5 \\
\hline 9. Se você precisa tomar remédios, isto atrapalha o seu dia-a-dia? & 0 & 1 & 2 & 3 & 4 & 5 \\
\hline 10. Volta líquido ou alimento do estômago em direção à boca? & 0 & 1 & 2 & 3 & 4 & 5 \\
\hline 11. Qual o grau de satisfação com a sua situação atual? & $\begin{array}{c}\text { muito } \\
\text { satisfeito }\end{array}$ & satisfeito & neutro & insatisfeito & $\begin{array}{c}\text { muito } \\
\text { insatisfeito }\end{array}$ & Incapacitado \\
\hline
\end{tabular}

0 não sinto; 1 sinto mas não me incomoda; 2 sinto e me incomoda, mas não todos os dias; 3 sinto e me incomoda todos os dias; 4 sinto e isto atrapalha o que eu faço durante o dia; 5 sinto e os sintomas não me deixam fazer nada.

TABELA 1 - Características gerais dos pacientes $(\mathrm{n}=124)$

\begin{tabular}{lc}
\hline Idade - média \pm DP & $42,6 \pm 13,5$ \\
\hline Sexo & $\mathrm{n}(\%)$ \\
$\quad$ feminino & $75(60,5)$ \\
$\quad$ masculino & $49(39,5)$ \\
Sintomas* & \\
$\quad$ pirose & $107(86,3)$ \\
regurgitação & $79(63,7)$ \\
disfagia & $21(16,9)$ \\
pigarro, rouquidão & $31(25,0)$ \\
dor torácica & $13(10,5)$ \\
tosse crônica & $27(21,8)$ \\
asma & $10(8,1)$ \\
\hline
\end{tabular}

* = vários sintomas podem apresentar-se em alguns pacientes

Avaliação da reprodutibilidade

Os escores do QS-DRGE respondidos pelos 10 pacientes estão na Tabela 2.

TABELA 2 - Características dos pacientes e escores inicial e final do QSDRGE $(\mathrm{n}=10)$

\begin{tabular}{llccc}
\hline Paciente (sexo/idade) & $\begin{array}{l}\text { Sintomas } \\
\text { (principal/secundário) }\end{array}$ & $\begin{array}{c}\text { Escore } \\
\text { inicial }\end{array}$ & $\begin{array}{c}\text { Escore } \\
\text { final }\end{array}$ & Diferença \\
\hline SR/feminino/ 38 & Pirose/regurgitação & 17 & 12 & -5 \\
PD/masculino/ 27 & Pirose/regurgitação & 13 & 14 & +1 \\
OPB/feminino/ 57 & Disfagia/pirose & 24 & 25 & +1 \\
NLGF/feminino/ 41 & Pirose/halitose & 19 & 19 & 0 \\
MTB/feminino/ 41 & Pirose/regurgitação & 14 & 12 & -2 \\
SW/feminino/ 33 & Regurgitação/pirose & 15 & 18 & +3 \\
GFF/masculino/ 30 & Regurgitação/pirose & 23 & 29 & +6 \\
ARBT/feminino/ 29 & Regurgitação/tosse & 17 & 21 & +4 \\
MR/masculino/ 59 & Tosse/pirose & 21 & 26 & +5 \\
OJB/masculino/ 38 & Pirose/rouquidão & 12 & 16 & +4 \\
\hline Média \pm DP & & & & \\
\multicolumn{1}{c}{ idade: $39,3 \pm 11$} & & $17,5 \pm 4,2$ & $19,2 \pm 5,9$ & $*$ \\
\hline
\end{tabular}

* Média da diferença: $1,7 \pm 3,4($ IC: $-5,0$ a 8,4$)$

A amostra foi composta por 10 pacientes e o coeficiente de correlação intra-classe obtido foi elevado, atingindo o valor de 0,833 devido à média da diferença entre as duas respostas.

\section{Coeficiente de correlação entre o escore QS-DRGE e o de Johnson-DeMeester}

A comparação dos dados obtidos entre o escore do QS-DRGE com os escores compostos de Johnson-DeMeester não evidenciou correlação entre as duas variáveis $(r=-0,03)$ (Figura 1$)$.

Os valores dos escores correspondentes aos 124 pacientes estão listados na Tabela 3.

\section{DISCUSSÃO}

$\mathrm{O}$ uso de instrumentos que permitam medir graus variados de "bem-estar" ou "mal-estar" pelos pacientes, tem permitido melhor compreensão sobre o impacto de diferentes doenças na qualidade de vida, como percebida pelos pacientes. Esses instrumentos, estruturados em forma de questionários, mensuram os assim chamados “domínios da saúde" ${ }^{(9)}$, quer em relação à qualidade de vida num aspecto geral, através dos chamados questionários genéricos, quer através de questionários específicos para determinadas enfermidades ${ }^{(5)}$.

O uso de um questionário estruturado, simples e com rápida execução e que possibilite o cálculo de escore que permita comparar um indivíduo ou grupo de indivíduos com outros e consigo próprio, ao longo de um período de tempo, tem enorme aplicação clínica, principalmente, após determinadas intervenções terapêuticas como, por exemplo, as farmacológicas ou cirúrgicas ${ }^{(2,10)}$. Estudo publicado por VELANOVICH et al. ${ }^{(12)}$ avaliou o efeito da síndrome da dor crônica (SDC) e das doenças psicoemocionais (DPE) na qualidade de vida de pacientes submetidos a cirurgia anti-refluxo,

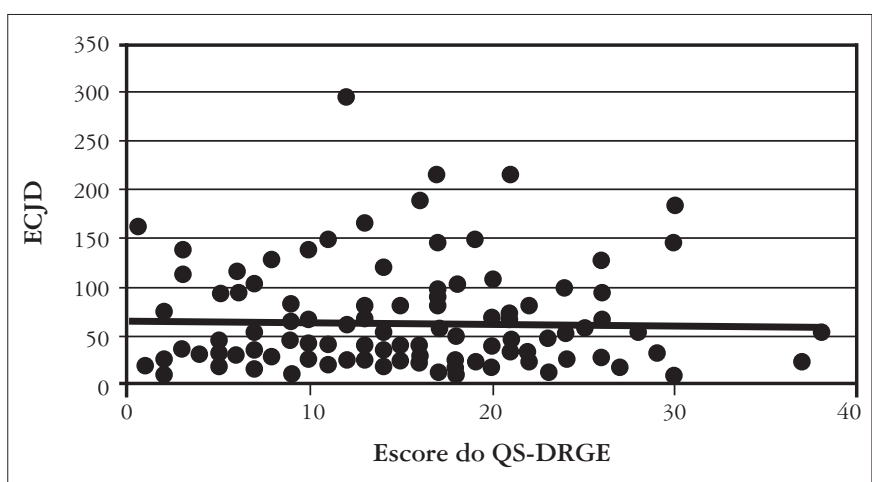

FIGURA 1 - Escore composto de Johnson e DeMeester (ECJD) comparado com o do QS-DRGE

TABELA 3 - Escores do QS-DRGE e composto de Johnson e DeMeester $(\mathrm{n}=124)$

\begin{tabular}{lcc}
\hline & Média \pm DP & Valores mínimo e máximo \\
\hline Escore do QS-DRGE & $13,41 \pm 6,98$ & $1-38$ \\
ECJD & $64,12 \pm 50,97$ & $8-297,1$ \\
\hline
\end{tabular}


utilizando a sua própria escala original de sintomas para a DRGE e o questionário de qualidade de vida genérico "SF-36". Após a cirurgia, os escores do questionário doença específico e os do SF-36 melhoraram significativamente tanto para o subgrupo de pacientes com SDC/PDE, quanto para o subgrupo (controle) sem esses distúrbios. Esses achados reforçam a utilidade tanto dos questionários doença-específicos, quanto a dos questionários genéricos para medir efeitos de intervenção, no caso cirúrgica, mesmo na ausência de distúrbios emocionais.

O questionário escolhido como referência para este trabalho, escrito originalmente na língua inglesa e validado numa amostra da população norte-americana para indivíduos com DRGE, tem sido reconhecido devido a sua fácil compreensão e aplicação em diferentes grupos de indivíduos. A construção do questionário QS-DRGE na língua portuguesa, compreendendo-se a tradução daquele e a sua adaptação aos vocábulos mais universais possíveis na língua portuguesa, para cada pergunta, foi bastante elaborada pelos autores e recebeu importante contribuição de um painel multidisciplinar de profissionais.

É importante salientar que VELANOVICH et al. ${ }^{(10)}$ construíram este questionário objetivando um instrumento de uso simples, fácil de ser entendido, mas com sensibilidade aos efeitos do tratamento. As questões submetidas aos pacientes foram fundamentadas em sintomas representativos da DRGE, exceto o último item, que avalia a satisfação ou percepção geral em relação à saúde pelo próprio paciente.

Questionários de qualidade de vida relacionados à saúde são construídos para transformar a avaliação subjetiva referente aos domínios da saúde, em escores numéricos correspondentes a cada domínio ou a um escore total ${ }^{(9)}$. Um questionário de qualidade de vida para a DRGE deveria contemplar o efeito da doença sobre diversos domínios como o físico, o psicológico e o social da saúde. No presente trabalho de tradução, adaptação e validação do original de Velanovich ao português, conclui-se que esse instrumento, ao conter apenas uma única questão especificamente sobre qualidade de vida, seria mais adequadamente denominado como "questionário de sintomas".

$\mathrm{O}$ original em inglês não continha questão sobre o sintoma "regurgitação", considerado como queixa típica e freqüente nos portadores de $\mathrm{DRGE}^{(8)}$. Portanto, decidiu-se acrescentar uma questão que mensurasse a presença de regurgitação, tornando o questionário na língua portuguesa mais abrangente, mas mantendo a sua concisão. Novo acréscimo de outras questões para mensurar a freqüência dos sintomas da DRGE ou outros poderia aumentar o tempo para o seu preenchimento. O questionário, dessa forma, continuou ocupando apenas uma única página e a questão acrescentada não gerou problemas de compreensão pelos pacientes, nem desfigurou o original.

Houve, no início do estudo, a preocupação com a compreensão e o tempo de preenchimento do questionário por parte de pacientes com diferentes níveis de escolaridade e que poderia truncar as respostas do questionário na forma auto-aplicável. Observou-se, eventualmente, dificuldade de interpretação das questões e das opções de respostas, em alguns indivíduos. Nessas circunstâncias os autores esclareceram as dúvidas, procurando a não-indução a quaisquer respostas. Surpreendentemente, o questionário foi respondido por todos os pacientes e o tempo gasto para tal foi, invariavelmente, menor que 5 minutos. O teste de reprodutibilidade evidenciou pequena variabilidade das respostas em dois momentos distintos, com um coeficiente de correlação intraclasse considerado excelente $(0,833)$, reforçando a validade do instrumento.
Ao comparar-se o escore do QS-DRGE com o composto de Johnson-DeMeester, entretanto, não se observou correlação. Esse fato foi também observado por VELANOVICH et al. ${ }^{(10)}$, no seu estudo clássico. Analisou-se, também, o grupo de pacientes cuja pHmetria esofagiana prolongada não foi compatível com refluxo gastroesofágico ácido patológico e verificou-se correlação nula entre os escores do QSDRGE e os de Johnson e DeMeester. Sabe-se que a correlação entre os sintomas da DRGE e a intensidade do refluxo gastroesofágico é pobre ${ }^{(13)}$. Possivelmente, a variabilidade individual quanto à sensibilidade ou à percepção do esôfago ou dos órgãos adjacentes ao refluxo ácido possam ser fatores de confusão e contribuam para a ausência de correlação.

Entretanto, a fácil compreensão pelos pacientes das perguntas do presente questionário, a rapidez no seu preenchimento e sua reprodutibilidade sugerem a sua utilidade na avaliação antes e após estudos com intervenção farmacológica, endoscópica ou cirúrgica na DRGE em língua portuguesa.

Em artigo de revisão sobre qualidade de vida, YACAVONE et al. ${ }^{(16)}$ descreveram e comentaram questionários disponíveis para o uso em pacientes com enfermidades gastroenterológicas e submetidos ao processo de validação. Para a DRGE o questionário "Qualidade de vida no refluxo e na dispepsia", descrito por WIKLUND et al. ${ }^{(15)}$, composto pelos domínios emoção, vitalidade, sono, alimentação e comportamento psicológico/social, direcionados a pacientes com dispepsia e/ou sintomas de refluxo gastroesofágico, enquanto que o "Questionário de qualidade de vida na doença péptica", de BAMFI et al. (1), compreendendo os domínios ansiedade induzida pela dor, limitação social e percepção de sintomas, para uso tanto em pacientes com úlcera péptica, esofagite ou dispepsia funcional. A limitação desses instrumentos é, basicamente, a sua construção e validação em um grupo de pacientes com doenças heterogêneas. A "Escala de qualidade de vida relacionada à saúde na doença do refluxo gastroesofágico", de VELANOVICH et al. ${ }^{(10)}$, apesar de falhar na abrangência de outros sintomas da DRGE, permite através da percepção do próprio indivíduo, discriminar pacientes satisfeitos ou insatisfeitos com sua situação de saúde após intervenção cirúrgica.

A maioria dos pacientes submetidos simultaneamente ao questionário de Velanovich e ao "SF-36" elegeu a escala de qualidade de vida relacionada à saúde na DRGE como mais fácil e compatível com os seus sintomas ${ }^{(11)}$.

Recente estudo publicado por JOHNSON et al. ${ }^{(7)}$, aplicando o questionário de Velanovich a pacientes submetidos ao implante endoscópico de Enteryx ${ }^{\circledR}$ para tratamento da DRGE, observou significativa redução dos escores relacionados ao sintoma pirose após 12 meses do implante e redução significativa do escore referente ao domínio físico da saúde avaliado pelo questionário SF-36. Importante limitação do presente instrumento é sua não abrangência dos chamados sintomas atípicos ou extra-esofágicos, cuja prevalência tem passado a ser cada vez mais reconhecida ${ }^{(8)}$

\section{CONCLUSÃO}

No presente estudo, o questionário de sintomas para a doença do refluxo gastresofágico, o QS-DRGE, apresentou validade de face, excelente reprodutibilidade, foi de fácil compreensão e rapidamente respondido. É necessário testar o presente questionário na língua portuguesa em diferentes grupos populacionais para aferir sua validade externa à presente pesquisa. 
Fornari F, Gruber AC, Lopes AB, Cecchetti D, Barros SGS. Symptom's questionnaire for gastroesophageal reflux disease. Arq Gastroenterol 2004;41(4):263-7. ABSTRACT - Background - Structured questionnaires are valuable instruments to measure the impact of specific diseases in patient's quality of life through a score and they are available such abroad as in Brazil. Nevertheless, questionnaires based on gastroesophageal reflux disease symptoms are not available in Portuguese. Aim - To develop and validate in Portuguese a specific questionnaire for gastroesophageal reflux disease symptoms. Patients and Methods - Velanovich's original questionnaire was translated, one question about "regurgitation" symptom was included and the vocabulary was adjusted to be understood to the scholarity level of the analyzed population. The "face validity" to each question was evaluated by the members of a multidisciplinary panel and a symptom's questionnaire for gastroesophageal reflux disease was developed. The questionnaire was applied to patients with gastroesophageal reflux disease symptoms confirmed by prolonged $\mathrm{pH}$ esophageal monitoring. The reproducibility, the comprehension, the time spent to fill out the questionnaire and the correlation coefficient to Johnson-DeMeester's score were measured. Results - The "face validity" was considered satisfactory by the panel and the questionnaire was applied to 124 patients, consecutively. The comprehension of the questionnaire and the time less than 5 minutes to fill out them were observed in all patients $(100 \%)$. Reproducibility for 10 patients in two different occasions showed a high intra-class correlation coefficient of 0,833 . The correlation to the Johnson-DeMeester's score was null. Conclusion - This study showed that the symptom's questionnaire for gastroesophageal reflux disease has "face validity", excellent reproducibility, easy comprehension and was quickly answered by patients. The correlation with Johnson-DeMeester's score was null.

HEADINGS - Gastroesophageal reflux. Signs and symptoms, digestive. Questionnaires.

\section{REFERÊNCIAS BIBLIOGRÁFICAS}

1. Bamfi F, Olivieri A, Arpinelli F, De Carli G, Recchia G, Gandolfi L, Norberto L, Pacini F, Surrenti C, Irvine SH, Apolone G. Measuring quality of life in dyspeptic patients. Development and validation of a new specific health status questionnaire. Am J Gastroenterol 1999;94:730-8.

2. Ben-Meier A, Urbach DR, Khajanchee YS, Hansen PD, Swanstrom LL. Quality of life before and after laparoscopic Heller myotomy for achalasia. Am J Surg 2001;181:471-4.

3. Ciconelli RM, Ferraz MB, Santos W, Meinão I, Quaresma MR. Tradução para a língua portuguesa e validação do questionário genérico de avaliação de qualidade de vida SF-36 (Brasil SF-36). Rev Bras Reumatol 1999;39:143-50.

4. Eisen GM, Locke GR, Provenzale D. Health-related quality of life: a primer for gastroenterologists. Am J Gastroenterol 1999;94:2017-21.

5. Guayatt GH, Freeny DH, Patrick DL. Measuring health-related quality of life. Ann Intern Med 1993;118:622-9.

6. Johnson DA, Ganz R, Aisenberg J, Cohen LB, Devière J, Foley TR, Haber GB, Peters JH, Lehman GA. Endoscopic implantation of Enteryx for treatment of GERD: 12-month results of a prospective, multicenter trial. Am J Gastroenterol 2003;98:1921-30.

7. Johnson LF, DeMeester TR. Twenty-four $\mathrm{pH}$ monitoring of the distal esophagus: a quantitative measure of gastro-esophageal reflux. Am J Gastroenterol 1974;62:325-32.

8. Moraes-Filho JPP, Cecconello I, Gama-Rodrigues J, Castro LP, Henry MA, Meneghelli UG, Quigley E, Brazilian Consensus Group. Brazilian consensus on gastroesophageal reflux disease: proposals for assessment, classification, and management. Am J Gastroenterol 2002;97:241-8.

9. Testa MA, Simonson DC. Assessment of quality-of-life outcomes. N Engl J Med 1996;334:835-40
10. Velanovich V, Vallance ST, Gusz JR, Tapia FV, Harkabus MA. Quality of life scale for gastroesophageal reflux disease. J Am Col Surg 1996;183:217-24.

11. Velanovich V. Comparison of generic (SF-36) vs. disease-specific (GERD-HRQL) quality-of-life scales for gastroesophageal reflux disease. J Gastrointest Surg 1998;2:141-5.

12. Velanovich $\mathrm{V}$. The effect of chronic pain syndromes and psychoemotional disorders on symptomatic and quality-of-life outcomes of antireflux surgery. J Gastrointest Surg 2003;7:53-8.

13. Venables TL, Newland RD, Patel AC, Hole J, Wilcock C, Turbitt ML. Omeprazole 10 milligrams once daily, omeprazole 20 milligrams once daily, or ranitidine 150 milligrams twice daily, evaluated as initial therapy for the relief of symptoms of gastro-oesophageal reflux disease in general practice. Scand J Gastroenterol 1997;32:965-73.

14. Ware JE Jr, Sherboune CD. The MOS 36-item short-form health survey (SF-36). I. Conceptual framework and item selection. Med Care 1992;30:473-83.

15. Wiklund IK, Junghard O, Grace E, Talley NJ, Kamm M, Veldhuyzen van Zanten S, Pare P, Chiba N, Leddin DS, Bigard MA, Colin R, Schoenfeld P. Quality of life in reflux, and dyspepsia patients. Psychometric documentation of a new disease-specific questionnaire (QOLRAD). Eur J Surg 1998;583(suppl):41-9.

16. Yacavone RF, Locke GR, Provenzale DT, Eisen GM. Quality of life measurement in gastroenterology: what is available? Am J Gastroenterol 2001;96:285-97. 